\title{
Assessment of Pattern, Severity and Outcome of Poisoning in Emergency Care Unit
}

\author{
Eswaran Maheswari $^{1 *}$, Lydia Abraham ${ }^{2}$, Chinju Susan Chacko², Ganesan Rajalekshmi Saraswathy ${ }^{1}$, \\ Aruna Chala Ramesh ${ }^{3}$ \\ ${ }^{1}$ Department of Pharmacy Practice, M.S. Ramaiah College of Pharmacy, Bangalore, Karnataka, India. \\ ${ }^{2}$ Department of Pharmacy Practice, Faculty of Pharmacy, M.S. Ramaiah University of Applied Sciences, Bangalore, Karnataka, India. \\ ${ }^{3}$ Department of Emergency Medicine, M.S. Ramaiah Hospitals, Bangalore, Karnataka, India.
}

\begin{tabular}{|c|c|}
\hline ARTICLE INFO & ABSTRACT \\
\hline Article history: & \multirow{10}{*}{$\begin{array}{l}\text { The current study was a prospective observational study. All poisoning cases admitted in the emergency care } \\
\text { unit of M.S. Ramaiah Hospital and Memorial Hospital were included in the study. A total of } 101 \text { patients were } \\
\text { included in the study. The pattern, severity and outcome of poison case were assessed using suitable scales. The } \\
\text { common agent involved in poisoning was drugs. It accounted for } 38.60 \% \text { of total poisoning cases. This was } \\
\text { followed by organophosphorous (OP) compounds, corrosive agents, rat poison, bedbug solutions and } \\
\text { insecticides. According to APACHE II the estimated mortality was found to be } 10.71 \text {. The mean Glasgow coma } \\
\text { scale was } 13.75 \pm 2.25 \text {. Out of } 101 \text { patients, } 100(99.01 \%) \text { recovered and } 1(0.99 \%) \text { died. Analysis of } 101 \text { poison } \\
\text { cases revealed that most poisonings are due to over dose of drugs and also due to consumption of OP } \\
\text { compounds. Establishment of strict policies against the sale and availability of agriculture field products and } \\
\text { over the counter drugs are an effective way to control OP and drug poisoning. Accidental poisoning by } \\
\text { paediatric groups can be minimized by conducting educational programs for the population in the rural regions. }\end{array}$} \\
\hline 2016 & \\
\hline Revis & \\
\hline Acce & \\
\hline Available o & \\
\hline Key words: & \\
\hline Poison; Severity, & \\
\hline Organophosphorous, & \\
\hline Glasgow coma scale, & \\
\hline & \\
\hline
\end{tabular}

\section{INTRODUCTION}

The word poison originates from the latin word potionem which means deadly draught. The Herald of modern Toxicology, Paracelsus, supposed that everything is poison and only the dose plays a pivotal role (Rajanandh et al., 2013). Any substance when ingested in large quantities can be toxic (Chiu et $a l ., 2011)$. Poison may be defined as any agent that can injure, kill or impair normal physiological function in humans producing general or local damage or dysfunction in the body by its chemical activity (Hakim et al., 2014). Poisoning occurs by the absorption of chemical, physical, or organic substances into the body through the gastrointestinal tract, skin, mucosa or respiratory tract or parentally causing damage to the cell, tissue and organs (Kara et al., 2014). Poisoning is a common medicosocial problem all over the world which may result in morbidity and mortality (Mar et al., 2008). In day to day life, knowingly

* Corresponding Author

Email: maheswarieswar @ gmail.com or unknowingly, millions of people are exposed to dangerous poisonous agents due to their unsafe storage and accessibility (Kiran et al., 2012). Poisoning is the fourth most common cause of death in India and it has been estimated that, five to six persons per lakh of population die due to acute poisoning every year (Susic et al., 2010). Knowledge of the epidemiology of poisoning and its changes is important to both emergency physicians and public health practitioners, but the treatment of poisoning can vary rapidly (Lee et al., 2008). Hence, regional epidemiological data on poisoning are very helpful in planning rational use of resources for the prevention and management of poisoning and in targeting research (Kavalci et al., 2008). The reasons most commonly stated for the large number of self-harm deaths relate to mental illness associated with war, poverty, unmet expectations, changing or breaking down of local cultures, chronic disease states, business loss, love failure or differences seen with the intimate partner, examination or emotional disturbances (Eddleston et al., 2006). In developed countries, it has been shown that the leading cause for visits to the emergency department among patients aged between 2 
to 30 years is acute poisoning, whereas in developing countries, it is the second most common cause following infectious disease (Carter et al., 2005).

Worldwide, poisonings account for $5-10 \%$ of all interventions (Susic et al., 2010). According to the World Health Organisation (WHO) more than 3 million poisoning cases with $2,51,881$ deaths occur worldwide annually of which, $99 \%$ of the fatal cases occur in developing countries (Hakim et al., 2014). Another estimate by WHO indicates that there may be 1 million serious unintentional poisonings each year and in addition 2 million people are hospitalised for suicidal attempts with pesticides (Parvin et al., 2011).Of the 1.87 lakh people who committed suicide in India in 2010, around 50 percent (49\% men and $44 \%$ women) are due to poison consumption, mainly pesticide (Kumar et al., 2010).In advanced countries, it has been observed that poisoning deaths are mainly due to consumption of cleansing agents, detergents, paracetamol, carbon monoxide and cosmetic products (Eddleston et al., 2006).Case fatality ratio (CFR) differs markedly between industrialized and developing worlds (Singh and Guntheki,2011). In India, poisoning rate varies between 15\% $30 \%$ of CFR (Shmertmann et al., 2008).

It is believed that between 7,50,000 and 30,00,000 OP poisoning cases occur globally every year. Mortality is higher in the developing countries where OP pesticides are readily available and may be misused for suicide. They are estimated to cause 3,00,000 fatalities annually (Kiran et al., 2004). Poisoning accounts for 1-6\% of bed occupancy in paediatric hospitals and $3.9 \%$ in paediatric intensive care unit in India. Poisoning is predominantly accidental particularly in age less than 5 years but might be increasingly self-inflicted in older children (Ketis et al., 2010). Lack of specialized toxicological services in developing countries like India has further contributed to the higher rates of morbidity and mortality (Kiran et al., 2012). Toxicology studies enabled for better understanding on the various poisonous agents (Langford et al., 2003). Pharmacokinetic and pharmacodynamic principles should be considered in the assessment and proper management of patients exposed to a poison. Clinicians must apply these principles to make rational clinical decisions regarding the significance of the poisoning (risk assessment) and to formulate an appropriate management plan (Khan et al., 2013). A better understanding of pharmacokinetic principles could improve the clinical care of patients (Abhubakar et al., 2014). Many specific poisoning treatments aim to favourably alter the pharmacokinetics of the poison, these include activated charcoal, whole bowel irrigation, extracorporeal elimination, chelating agents, antitoxins and urinary alkalinisation (Roberts and Buckley, 2007). The common patterns of poisoning are suicidal, homicidal/criminal and accidental. The incidence, nature, aetiology, age group affected and the outcome of poisoning in India is different from that of the western world (Anthony and Kulkarni, 2012).

The cornerstone of treatment includes supportive measures, timely gastrointestinal (GI) decontamination, extracorporeal methods of elimination of poisons and antidotes
(Kara et al., 2014). It is therefore important to know the nature and severity of poisoning to take prompt measures in saving life and thereby reduce morbidity and mortality (Hakim et al., 2014). Also it is important in applying appropriate techniques for diagnosis and thereby improving one's quality of life (Acharya et al., 2014). Early management and preventive strategies are crucial in reducing the burden of poisoning and related injuries (Eddleston et al., 2006). Management of poisoned patients will greatly improve, if the common causes of poisoning are properly defined. Knowledge of general pattern of poisoning in a particular region will help in early diagnosis and treatment of cases, thus decreasing the rate of mortality and morbidity (Karikalan and Murugan, 2014).It is important to know the nature and severity of poisoning in order to take appropriate preventive measures.Hence, studies of this nature will remain as a useful tool in planning and managing critically ill acute poisoning cases. It also helps in framing appropriate policies like introducing new guidelines and updating prevailing treatment protocols, counselling and sensitizing the society on hazards of poisoning and proper usage and storage of chemicals (Aravind and Rai, 2014). The current study was undertaken to assess the pattern and determinants of poisoning in Emergency Care unit and to assess the severity and outcome of poisoning.

\section{MATERIALS AND METHODS}

\section{Study design and setting}

The study was a prospective observational study carried out at the Department of Accident and Emergency in M.S. Ramaiah Hospital and Memorial Hospital, Bangalore, Karnataka for a period of 6 months from January to June 2015. The data was collected from patient case sheets using data collection forms. All poisoning cases admitted in the emergency care unit of M.S. Ramaiah Hospital were included in the study. Patients with comorbid conditions including metabolic causes and structural brain related causes and those who were unwilling to give informed consent were excluded from the study. A total of 101 patients were included in the study based on the study criteria.

\section{Ethical approval}

Ethical approval was obtained from Institutional Ethics Committee of M.S. Ramaiah Medical College, Bangalore, India.

\section{Data analysis}

All the quantitative variables in the present study such as age, gender, number of days stayed in the hospital etc., have been summarised in terms of mean, standard deviation, median. The statistical software SPSS 20.0 was used for data analysis.

\section{Study instrument}

The study included Glasgow coma scale (GCS), Poisoning severity score, and Simplified Acute Physiology Score II (SAPSII) for the analysis of severity and outcome of poisoning. Glasgow Coma Scale is used for the assessment of severity of illness in non-traumatic patients. The eye, motor and verbal 
responses will be analysed and scored from comatose to stable state i.e from 1 to 15 (Nikhita et al., 2015). The Poison Severity Score takes into account the overall clinical course and is applied according to the most severe symptomatology. This study tool helps in analysing various organ systems and their functions and further scorings will be made as none, minor, moderate, severe and fatal(Poison Severity Score, 1998).The Simplified Acute Physiology Score II is made of 12 physiological variables and 3 disease related variables. The worst physiological parameters were collected during the initial 24 hours duration and the scorings ranges from 0 to 163 . The various parameters included are age, heart rate, systolic blood pressure, temperature, GCS, urine output, blood urea nitrogen, serum sodium, serum potassium, serum bicarbonate, serum bilirubin, white blood cell count and comorbidity (Prakash et al., 2006).

\section{Study procedure}

The study was done in the Department of Emergency Medicine of M.S. Ramaiah Hospital. All the poisoning cases admitted to the department during the study period were documented. Using a suitably designed data collection form, the required information such as patient demographics, state of consciousness, time of admission, determinants of poisoning, initial treatment given and antidoteadministered were collected from the patient case file, nursing notes and medication charts. The pattern of drugs used for self-poisoning and its consequences on morbidity was studied. The pattern and severity of poison case was then assessed using suitable scales. The outcomes of the treatment given were also reported.

\section{RESULTS}

The study was conducted over a period of 6 months in M.S. Ramaiah hospital and Memorial hospital. A total of 101 patients were observed and the following evaluations were made.

\section{Age category}

The age group of the patients were from 1-70 years. Of which most of the cases were in the age group of 21-30 years. Least were found in 61-70 years. The mean age of the study population was found to be $27.60 \pm 12$.66years. The number of patients based on age category is specified in Table 1 .

Table 1: Frequency table for age in the study population.

\begin{tabular}{ccc}
\hline Age & Number of Patients (n) & Percentage (\%) \\
\hline $1-10$ & 10 & 9.9 \\
$11-20$ & 13 & 12.9 \\
$21-30$ & 46 & 45.5 \\
$31-40$ & 18 & 17.8 \\
$41-50$ & 7 & 6.9 \\
$51-60$ & 5 & 5 \\
$61-70$ & 2 & 2 \\
\hline
\end{tabular}

\section{Gender distribution and marital status}

The numbers of female patient were 57 (58.7\%) slightly higher than males $44(43.6 \%)$. Of the total patients $54.4 \%$ were married and $45.5 \%$ were unmarried. The details about distribution of gender and marital status are provided in Table 2.

Table 2: Distribution of gender and marital status.

\begin{tabular}{lccc}
\hline \multicolumn{1}{c}{ Gender } & Percentage (\%) & Married & Unmarried \\
\hline Male & 43.6 & 22.77 & 20.79 \\
Female & 58.7 & 31.68 & 24.75 \\
\hline
\end{tabular}

\section{Mode of poisoning}

It was found that $79.2 \% \quad(n=80)$ of the cases were intentional poisoning for suicidal attempt and $20.8 \%(n=21)$ of the cases were accidental poisoning. The details about gender,age pattern and mode of poisoning are provided in Table 3 .

Table 3. Distribution gender,age pattern and mode of poisoning

\begin{tabular}{clcc}
\hline \multicolumn{2}{c}{ Mode of poisoning } & Suicidal $\%$ & Accidental $\%$ \\
\hline \multirow{2}{*}{ Gender } & Male & 36.64 & 7.92 \\
\cline { 2 - 4 } & Female & 43.56 & 12.87 \\
\hline \multirow{3}{*}{ Age } & $1-10$ & 0.99 & 8.91 \\
\cline { 2 - 4 } & $11-20$ & 9.90 & 2.97 \\
\cline { 2 - 4 } & $21-30$ & 37.62 & 7.92 \\
\cline { 2 - 4 } & $31-40$ & 16.83 & 0.99 \\
\cline { 2 - 4 } & $41-50$ & 6.93 & 0 \\
\cline { 2 - 4 } & $51-60$ & 4.93 & 0 \\
\cline { 2 - 4 } & $61-70$ & 1.98 & 0 \\
\hline
\end{tabular}

\section{Hospital stay of the patients}

Among 101 patients, $91.1 \%$ of patients stayed in the hospital for 1-2 days, $5.9 \%$ for 2-4 days and 3\% for 4-6 days. The mean hospital stay was found to be $1.1 \pm 0.42$ days and the median was 24 hours.

\section{Type of poisoning}

The common agent involved in poisoning was drugs, which accounted for $(38.6 \%)$ of total poisoning cases and least was bedbug solution which accounted for (4.9\%) of the total poisoning case. The type of poison consumed by patients is specified in Table 4.

Table 4: Distribution of various types of poison.

\begin{tabular}{lcc}
\hline \multicolumn{1}{c}{ Type of poison } & $\begin{array}{c}\text { Number of } \\
\text { patients }(\mathbf{n})\end{array}$ & $\begin{array}{c}\text { Percentage } \\
(\boldsymbol{\%})\end{array}$ \\
\hline Organophosphorous compounds & 23 & 22.7 \\
Rat poison & 6 & 5.9 \\
Corrosive agents & 17 & 16.8 \\
Bed Bug Solution & 5 & 4.9 \\
Drugs & 39 & 38.6 \\
Unknown & 11 & 10.8 \\
\hline
\end{tabular}

Table 5: Distribution of drugs for poisoning.

\begin{tabular}{lcc}
\hline \multicolumn{1}{c}{ Category of Medicine } & $\begin{array}{c}\text { Number of patients } \\
(\mathbf{n})\end{array}$ & Percentage (\%) \\
\hline Antipsychotics & 23 & 58.98 \\
Antithyroid agents & 3 & 7.69 \\
Analgesics & 5 & 12.82 \\
Antihypertensives & 3 & 7.69 \\
Unknown Drugs & 5 & 12.82 \\
\hline
\end{tabular}

\section{Drugs involved in poison}

Of all the cases of poisoning with medicines, psychiatric drugs accounted for $58.98 \%$ and least was with antithyroid agents 
and antihypertensives. The category of drugs consumed by patients is provided in Table 5 .

\section{Types of organophosphorous poisoning}

The overall poisoning with OP compounds was found to be $22.7 \%$. Of the 23 cases of OP poisoning, 9 were due to pyrethrins $(39.1 \%), 1$ due to tick 20 spray (4.3\%). The type of organophosphorous compounds consumed by patients are listed in Table 6.

Table 6: Pattern of organophosphorous agents used for poisoning.

\begin{tabular}{lcc}
\hline $\begin{array}{c}\text { Category of } \\
\text { Organophosphorous } \\
\text { Compound }\end{array}$ & $\begin{array}{c}\text { Number of } \\
\text { Patients (n) }\end{array}$ & Percentage (\%) \\
\hline Pyrethrins & 9 & 39.1 \\
Carbamate & 5 & 21.73 \\
Tick 20 & 1 & 4.3 \\
Insecticides & 5 & 21.73 \\
Unknown & 3 & 13.12 \\
\hline
\end{tabular}

\section{Category of antidote}

Antidotes were administered for $26.7 \%$ of the cases. The use of antidotes was specific for the poisoning case. Atropine was used in $44.44 \%$ of the cases. The various antidotes used for poisoning is provided in Table 7 .

Table 7: Distribution of the various types of antidotes used for poisoning.

\begin{tabular}{lcc}
\hline Category of Antidote & $\begin{array}{c}\text { Number of } \\
\text { Patients(n) }\end{array}$ & Percentage (\%) \\
\hline Atropine & 10 & 44.44 \\
Flumazenil & 9 & 37.03 \\
Sodium bicarbonate & 3 & 11.11 \\
N-acetylcysteine & 1 & 3.70 \\
Chelating agent & 1 & 3.70 \\
\hline
\end{tabular}

\section{Treatment}

Gastric decontamination by lavage and administration of activated charcoal are known to limit the absorption of some ingested poisons provided they are given within one-hour post ingestion and only if the airway is protected. $80.2 \%$ of patients had undergone gastric lavage. The pattern of treatment provided for poisoning is provided in Table 8 .

Table 8: Pattern of treatment provided for poisoning.

\begin{tabular}{lcc}
\hline \multicolumn{1}{c}{ Treatment } & $\begin{array}{c}\text { Number of } \\
\text { Patients (n) }\end{array}$ & Percentage (\%) \\
\hline Gastric lavage & 81 & 80.2 \\
Proton pump inhibitors & 93 & 92.1 \\
Antiemetics & 88 & 87.1 \\
Antidote & 27 & 26.7 \\
Normal saline & 35 & 34.7 \\
Dextrose normal saline & 6 & 5.9 \\
H2-Receptor blocker & 5 & 5.0 \\
\hline
\end{tabular}

\section{Assessment of severity}

\section{Glasgow Coma Scale scores}

Glasgow coma score was 11 and below in 3 patients, 13 and below in 5 patients. 29 patients $(38.7 \%)$ had a score of
15.Patients with score 11 and below were instituted several interventions like antidote, gastric lavage, oxygen supply and intravenous fluids. The mean Glasgow coma scale was 13.75 \pm 2.25 . The score obtained using Glasgow coma scale is provided in Table 9.

Table 9: Scores obtained by Glasgow Coma Scale.

\begin{tabular}{ccc}
\hline Score & Number of patients (n) & Percentage $(\%)$ \\
\hline 0 & 1 & 1.0 \\
3 & 2 & 2.0 \\
5 & 1 & 1.0 \\
11 & 1 & 1.0 \\
12 & 6 & 5.9 \\
13 & 22 & 21.8 \\
14 & 31 & 30.7 \\
15 & 37 & 36.6 \\
\hline
\end{tabular}

\section{Poison Severity Score}

$52.6 \%$ of the cases were classified as minor, $20 \%$ were classified as moderate and $27.3 \%$ were classified as none (not showing any symptoms). The poison severity score is provided in Table 10.

Table 10: Analysis using Poison Severity Score.

\begin{tabular}{cc}
\hline Severity & Percentage (\%) \\
\hline Minor & 52.6 \\
Moderate & 20.0 \\
None & 27.3 \\
\hline
\end{tabular}

\section{Simplified Acute Physiology II Score}

Simplified Acute Physiology II Score is found to be helpful in estimating severity and clinical prognosis of poisoning. The point score is calculated from 12 routine physiological measurements during the first 24 hours, information about previous health status and some information obtained at admission. In our study this scoring was done for only 22 patients,since only 22 patients were inpatients. The estimated mortality rate for the 22 patients was found to be 10.26 . The details about estimated mortality rate are provided in Table 11.

Table 11: Simplified Acute Physiology II Score estimated mortality rate.

\begin{tabular}{ccc}
\hline SAPS II & Number of patients (n) & Percentage (\%) \\
\hline $1-3$ & 2 & 9.10 \\
$4-6$ & 8 & 36.36 \\
$7-9$ & 1 & 4.54 \\
$10-12$ & 5 & 22.72 \\
$13-15$ & 1 & 4.54 \\
$16-18$ & 3 & 13.63 \\
$19-21$ & 2 & 9.09 \\
\hline
\end{tabular}

\section{Outcome of poisoning}

Out of 101 patients, $100(99.01 \%)$ recovered and 1 $(0.99 \%)$ died. The death of the patient was due to late admission to hospital after suicidal attempt with insecticide.

\section{DISCUSSION}

Poisoning was more frequently observed in younger age groups (21-30) than older age groups. The mean age of the case 
was observed to be $27.60 \pm 12.66$ years. This was similar to the findings of Grzinic et al., (2009) where the mean age of poisoned patients was $33.4 \pm 16.0$ years. Poisoning was found to be predominant in females $(56.4 \%)$ compared with males $(43.6 \%)$ in our study. There was preponderance of females presenting with poison exposure in a study conducted in Singapore enrolling 635 patients which was done by Chiu et al., (2011).

The median hospital stay in this study was 1 day which was comparable to the study done in Turkey by Kara et al., (2014) where 86 patients were enrolled for the study and the median hospital stay was found to be 1 day. $20 \%$ of cases were under the age of 20 years. This was similar to the study done by Susic et al., (2010) where 244 cases were included for the study and the percentage of cases below the age of 20 was in line with National Slovenian Register, but this is considerably lower than that in the United States. The highest number of cases were in 21-30 years of age $(45.5 \%)$ followed by $31-40$ years of age $(17.8 \%)$ and $11-20$ years of age (12.9\%). In the age group of 21-30 years, females were $29.7 \%$ and males were $15.84 \%$. It was similar to the findings of a study done in Karnataka by Ramesha et al., (2009) in which 136 patients were studied and $31.2 \%$ of cases presented in the age group between 20 and 29 years of which, 53\% was occupied by female and $47 \%$ was occupied by males.55\% of patients in the study were married and most of them were in their second decade of life. The study finding was in par with the study done by Acharya et al., (2014) where $71 \%$ of cases were married and $29 \%$ were unmarried. In the present study, we found that poisoning was more prevalent among married people compared to unmarried that may be because of exposure to a greater number of stressful situations and may also be attributed to each individuals capacity to handle stress.

Among the type of poisonings observed in this study $38.6 \%$ were due to consumption of drugs $(n=39), 22.7 \%$ OP $(n=23), 17 \%$ corrosives $(n=17), 16.8 \%$ rat poison $(n=6), 5.9 \%$ bed bug solution $(n=5), 5 \%$ and $5 \%$ with unknown substances $(n=11)$, $10.8 \%$. The study done by Acharya et al., (2014) showed similar findings where OP poisoning accounted for $58 \%$ of cases, corrosives $25 \%$, rat poison $6 \%$, drugs $5 \%$ and unknown $6 \%$.This pattern relates to the easy availability of the above compounds.

There were 4 different pharmacological groups that were involved. Psychiatric drugs were used by 23 patients $(58.98 \%)$ followed by levothyroxine $(n=3,7.69 \%)$, analgesics $(n=5$, $12.82 \%)$, antihypertensives $(n=3,7.69 \%)$ and unknown drugs $(\mathrm{n}=5,12.82 \%)$ which was on par with a study conducted by Jalali et al., (2012) where $31.6 \%$ of cases were due to consumption of medicines such as antidepressants (58\%) followed by antihypertensives $(11.4 \%)$ and unknown drugs $(30.6 \%)$. The main drug-groups causing poisoning were CNS-acting drugs. Regardless of the type of poisoning (intentional or accidental), drugs were involved in poisoning. Obeying the academic and standard principles for prescription and drug usage can prevent poisoning due to drugs to an extent.

OP poisoning accounted for $17.68 \%(n=18)$ of all the cases. It was found to be the second most common reason for poisoning in the current study. Similar results were found in a study conducted by Shaikh, et al.,(2011) in Liaquat University of Medical and Health Sciences where 100 patients were enrolled and OP compounds $(33 \%)$ were the second most common reason for poisoning following travel related poisoning $(57.56 \%)$. India being an agricultural based country, OP pesticide remains the main agent for crop protection and pest control. It is therefore likely to have adverse effects on farmers who are accidently over exposed while handling these pesticides. However, because of low cost and easy availability, it has also become an agent of choice for self poisoning.

This study showed that intentional poisoning for suicidal attempt was reported among 80 cases $(79.2 \%)$ compared to 21 cases $(20.8 \%)$ that were accidentally exposed to toxic substances. Ramesha et al., (2009) found that $77.9 \%(n=106)$ of cases were of suicidal intention and $22.1 \%(\mathrm{n}=30)$ of cases had accidental poisoning. Among the 80 suicidal admissions, percentage of males outweighed than females by $6.92 \%$. This high proportion of poisoning among males might be due to change in the lifestyle, cultural patterns in the area or emotional stress (Mar et al., 2008). Accidental poisoning $(8.91 \%)$ was more in children aged between 1-10 years compared to $0.99 \%$ of suicidal cases. Aggarwal et al., (2014) observed similar findings where 90 children were enrolled for the study and all the poisoning episodes were either suicidal $(n=38.46 .9 \%) \quad$ or accidental $\quad(n=43, \quad 53.1 \%)$.Children's accessibility of poison is influenced by socio-economic status, education, local beliefs' and customs of the community (Mar et al., 2008). Glasgow coma score was 11 in 3 patients and below 13 in 5 patients.29 patients $(38.7 \%)$ had a score of 15 . The mean score in our study was $13.75 \pm 2.25$. This was in par with the findings of a study done by Arvind et al., (2014) where the Glasgow scale of the patients on admission to intensive care unit ranged from 10 to 15 in $60 \%$ of cases. This indicates that the patients were conscious at the time of admission. The estimated mortality rate using APACHE II scoring was found to be 10.71 and SAPS II scoring was 10.26 . This was comparable to the results in the study done by Nikhita et al., (2015) in Bangalore. The analysis using Poison Severity Score yielded $52.6 \%$ of cases classified as minor, $20 \%$ cases as moderate and $27.3 \%$ cases without any symptoms. In our study, 100 patients $(99.01 \%)$ recovered and 1 died $(0.99 \%)$. Death was reported for the patient of 46 years who has died due to late admission to the hospital after suicidal attempt with insecticides. Similar finding was observed in the study done by Nikhita et al., (2015) where out of 90 patients 1 death was reported.

\section{CONCLUSION}

Most poisoning are by over dose of drugs and also due to OP compounds. The incidence rate was high in the age group of 21-30 years. Establishment of strict policies against the sale and availability of agriculture field products and over the counter drugs are an effective way to control OP and drug poisoning. In our region, intoxication for suicidal attempts is common in young females. The reason for poisoning among majority of the patient 
population might be family problems which cannot be treated medically. But providing awareness, counselling and appropriate supportive care can decrease the occurrence of poisoning in suicidal cases to prevent further attempts in future. Accidental poisoning by paediatric groups can be minimized by conducting educational programs for the population in the rural regions.

\section{ACKNOWLEDGEMENT}

We sincerely thank our Principal, Dr. V. Madhavan for giving us an opportunity and providing us the resources and support during our project work.

\section{Financial support and sponsorship: Nil.}

Conflict of Interests: There are no conflicts of interest.

\section{REFERENCES}

Abubakar S, Githa K, KiranN. A study on pattern of poisoning cases in a tertiary care hospital, Bangalore.Indian Journal of Pharmacy Practice, 2014; 7(1):13-17.

Acharya S, Lakshminarayana K, Sharanappa. Assessment of poisoning cases in a tertiary care hospital. IJBR, 2014; 5(9):578-581.

Aggarwal B, Rana SK, Chhavi N. Pattern of poisoning in children, an experience from a teaching hospital in northern India. JK Science, 2014; 16(4): 174-178.

Anthony L, KulkarniC. Patterns of poisoning and drug overdosage and their outcome among in-patients admitted to the emergency medicine department of a tertiary care hospital. Indian J Crit Care Med, 2012; 16(3):130-135.

Aravind A, Rai M. Pattern of acute poisoning admissions in the medical intensive care unit of a tertiary care hospital. Internal $\mathrm{J}$ of pharmaceutical sciences and drug research, 2014; 6(3): 239-242.

Carter G, Reith DM, Whyte IM, McphersonM. Repeated selfpoisoning: Increasing severity of self-harms a predictor of subsequent suicide. British J of Psychiatry, 2005; 18(6):253-257.

Chiu L, Lim B, Vasu A, PhuaDH. Poison exposure in the emergency department: a Singaporean experience. Hong Kong J. of Emerg Med, 2011; 18(4):97-202.

Eddleston M, Karunaratne A, Weerakoon M, Kumarasinghe S, Rajapakshe M.Choice of poison for intentional self-poisoning in rural Sri Lanka.ClinToxicol (Phila), 2006; 44(3):283-286.

EddlestonM,Sudharshan K, Senthilkumaran M. Patterns of hospital transfer for self-poisoned patients in rural Sri Lanka: Implications for estimating the incidence of self-poisoning in the developing world. Bulletin of the world health organization, 2006; 84(4):276-282.

GrzinicMK, Susic P, Marija B, Ketis K, Bunc T. Aetiological and demographical characteristics of acute poisoning in the Celje region, Slovenia. European J of Emerg Med, 2009; 16(3):127-130.

Hakim A, Khurshid R, Mufti S, Krishan K, Singh Y.Pattern, profile and outcome of poisoning cases: a study at a large teaching hospital in India. JK practitioner, 2014; 19(1-2): 36-40.

Hunter LJ,Wood DM, Dargan PI. The patterns of toxicity and management of acute nonsteroidal anti-inflammatory drug (NSAID) overdose. Open Access Emergency Medicine, 2011; 3:39-48.

Jalali A, Savari M, Dehdardargahi S, AzarapanahA.The pattern of poisoning in southwestern region of Iran: envenoming as the major cause. Jundishapur J Nat Pharm Prod, 2012; 7(3):100-105.

Kara H, Bayir A, Degirmenci S, Akinci M, Ahmet AK, Kayis SA. Causes of poisoning in patients evaluated in a hospital emergency department in Konya, Turkey. J Pak Med Assoc, 2014; 64(9):1042-1048.

Karikalan T, Murugan M. Profile of poisoning cases in a tertiary care hospital, Tamilnadu. J of Evolution of Med and Dent Sci,2014; 3(56):12754-12760.
Kavalci G, Ethemolglu FB, Kavalci C. Characteristics of poisoning cases in Ankara Turkey. J of Emerg Med, 2008; 5(1):17-19.

Ketis ZK,Susic TP, Grizinic KM, Kersnik J.Glasgow Coma Scale in Acute Poisonings before and after use of antidote in patients with history of use of psychotropic agents, GCS analysis. Srp Arh Celok Lek, 2010; 138(3-4):210-213.

Khan NA, Rahman A, Sumon SM.Pattern of poisoning in a tertiary level hospital.Mymensingh Med J,2013; 2(2):241-247.

Kiran N, Rani S, Jaiprakash V, VanajaK. Pattern of poisoning reported at south Indian tertiary care hospital.Ind $\mathrm{J}$ of Forensic Med and Toxicol,2012; 2(2):72-76.

Kumar A, Verma A, Jaiswal K, Kumar S, PrasadR. Emergency of entirely new poisoning in rural India, an upcoming health hazard to the community health. Indian J of community health,2012; 24(3):248-251.

Langford NJ, Liang GW, Baetman DN. Quinine intoxications reported to the Scottish Poisons Information Bureau 1997-2002: A continuing problem. J Clin Pharmacol, 2003; 56(2):576-578.

Lee LH, Lin HJ, Yeh STY, Chi CH. Presentations of patients of poisoning and predictors of poisoning-related fatality: Findings from a hospital-based prospective study. BMC Public Health, 2008; 8(7):1-9.

Mar H, Sardar MH, Awin MR, Morshed MG, Islam MS, AzharMA.Clinico-epidemiological pattern of poisoning in a tertiary level hospital. J Dhaka Med Coll, 2008; 17(2):111-115.

Nikhita D, Jasem DZ, Ramaswamy R, Royl AP, ReddyalAM. Assessment of severity of organophosphate poisoning at a tertiary care hospital, South India.World J of pharma research, 2015; 4(1):882-888.

Parvin R, Haque N, Ahmed N, Ali MI, Rafiqueuddin AKM. Pattern of poisoning cases in a tertiary hospital in Bangladesh. J Enam Med Col, 2011; 1(1):19-23.

Poison Severity Score. IPCS/EAPCCT Grading of acute poisoning. J Toxicology- clinical toxicology, 1998; 36:205-213.

Prakash P, Krishna K, Bhatia D. Usefulness of SAPSII scoring system as an early predictor of outcome in ICU patients. J Indian academy of clinical medicine, 2006; 7(3): 202-205.

Rajanandh MG,Santhosh S, Ramasamy C. Prospective analysis of poisoning cases in a super speciality hospital in India. $\mathrm{J}$ of pharmacology and toxicology,2013; 8(2):60-66.

Ramesha KN, Rao KBH, KumarGS. Pattern and outcome of acute poisoning cases in a tertiary care hospital in Karnataka, India. Indian J Crit Care Med, 2009; 13(3):152-155.

Roberts DM, BuckleyNA. Pharmacokinetic Considerations in Clinical Toxicology. Clin. Pharmacokinet, 2007; 46(11):899-907.

Schmertmann M, Williamson A, Black D. Stable age pattern supports role of development in unintentional childhood poisoning. Injury Prevention, 2008; 14:30-33.

ShaikhMA. Mortality in patients presenting with organophosphorus poisoning at Liaquat University of Medical and Health Sciences. Pak J Med Sci, 2011; 27(5):1022-1024.

Singh UP, Guntheti BK. The pattern of poisoning in khammam. J Indian Acad Forensic Med, 2011; 33(4):91-93.

Susic TP, Ketis ZK,Grzinic KM, Kersnik J. Glasgow Coma Scale in acute poisonings before and after use of antidote in patients with history of use of psychotropic agents. Srp Arh Celok Lek, 2010; 138(3-4): 210-213

How to cite this article:

Maheswari E, Abraham L, Chacko CS, Saraswathy GR, Ramesh AC. Assessment of Pattern, Severity and Outcome of Poisoning in Emergency Care Unit. J App Pharm Sci, 2016; 6 (12): 178-183. 